\title{
Décadrages Décadrages
}

cinéma, à travers champs Cinéma, à travers champs

\section{4-36 | 2017}

\section{Cinéma de re-montage}

\section{Une jeunesse allemande (Jean-Gabriel Périot) : qui parle?}

\section{François Albera}

\section{(2) OpenEdition}

1 Journals

Édition électronique

URL : https://journals.openedition.org/decadrages/1060

DOI : 10.4000/decadrages. 1060

ISSN : 2297-5977

Éditeur

Association Décadrages

Édition imprimée

Date de publication : 1 mai 2017

Pagination : 72-93

ISBN : 978-2-9700963-3-7

ISSN : 2235-7823

\section{Référence électronique}

François Albera, « Une jeunesse allemande (Jean-Gabriel Périot) : qui parle? », Décadrages [En ligne], 34-36 | 2017, mis en ligne le 19 août 2019, consulté le 04 avril 2022. URL : http://

journals.openedition.org/decadrages/1060; DOI : https://doi.org/10.4000/decadrages.1060

(B) Décadrages 
François Albera

\section{Une jeunesse allemande (Jean-Gabriel Périot): qui parle?*}

d'un article destiné à la revue Chimères (n 89 ) de février 2017 dont le dossier est consacré aux «Potentialités cinématographiques».

Une jeunesse allemande (2015) APPARTIENT À un genre cinématographique que l'on appelle, depuis les années 1920, «film de montage»: il utilise des matériaux filmés préexistants de nature documentaire et en propose un re-montage original. Cette pratique, inhérente en quelque sorte au cinéma (assemblages de «vues» Lumière sur un thème au sein d'un programme, puis de bandes d'actualité ou documentaires au sein d'un film plus vaste), a connu une mutation en accédant à une dimension proprement discursive en URSS avec, en particulier, Esfir Choub et Dziga Vertov. C'est surtout dans le milieu du cinéma militant des années i920 (notamment en Allemagne, aux Pays-Bas, en Belgique - avec entre autres Blum, Dudow, Storck) que cette pratique de la compilation de bandes d'actualités réorganisées dans une perspective donnée a connu une faveur, au croisement de nécessités économiques (difficultés financières et matérielles à constituer une agence d'actualités avec des opérateurs envoyés sur les lieux des événements) et idéologiques (volonté de «déconstruire» le propos des actualités des grandes maisons de production comme Pathé, Gaumont, Fox, UFA, etc.). On en a quelques exemples français dus notamment à Jacques-Bernard Brunius - membre de l'Association des écrivains et artistes révolutionnaires (AEAR), du Groupe Octobre, collaborateur de Jean Renoir -, avec ses films Records 37 (I937), Sources noires (1937), Violons d'Ingres (I937), ou à Jean-Paul Dreyfus et quelques autres. Et des exemples partiels dans des films comme Borinage (I933) ou La Vie est à nous (1936) qui comportent des séquences de ce type. Durant la Deuxième Guerre mondiale, la production étatique de propagande ou d'instruction (des militaires ou des populations) adopte en quelque sorte cette démarche et la banalise en lui ôtant sa dimension critique. Si l'on ne cesse pas de réaliser de tels montages - ainsi le fameux Paris I9oo (1945) de Nicole Védrès -, il faut attendre les années I960 pour retrouver des longs métrages de compilation ambitieux au plan historique tant en 
Allemagne avec Erwin Leiser (Mein Kampf, ı96o; Eichmann und das Dritte Reich, 196r), qu’en France avec Frédéric Rossif (Le Temps du ghetto, I96I, Mourir à Madrid, I963, La Révolution d'Octobre, I967), en Italie avec Lino Del Fra, Cecilia Mangini, Lino Micciché (All'armi siam fascisti, I962) ou en URSS, avec Le Fascisme ordinaire (1964) de Mikhaïl Romm. Il va sans dire que ce genre - qui va perdre rapidement à nouveau toute dimension critique - va fournir une partie importante de la production télévisuelle «historique» (comme les émissions «honnêtement» trompeuses d'Alain de Sédouy par exemple) jusqu'aux «superproductions» actuelles du type «Apocalypse» (cyniquement manipulatrices).

Ces dernières ont mis en lumière, plus encore que les films de cinéma, les problèmes que pose cette pratique dans l'usage des archives filmées - terme qui s'est substitué désormais à celui d'«actualités» (disparues des cinémas) et qui est souvent employé au singulier depuis Foucault et Derrida. L'intégration de matériaux préexistants à un nouvel ensemble qui impose sa narration visuelle et sonore - souvent coiffée d'un commentaire off explicatif et entrecoupée d'inserts avec des paroles d'experts en histoire ou en stratégie militaire, voire de témoins - se fait la plupart du temps sans référencement des sources utilisées, datations ou localisation. L'adjonction du commentaire, de sons ajoutés en studio (bruitage) ou de musiques d'accompagnement comme, plus récemment, de la colorisation, tend à obtenir et produit une homogénéité trompeuse qui efface les disparités des matériaux, évince les points de vue qui ont présidé aux prises de vue et mêle même volontiers sans le dire des images de fiction aux documents. La nécessité que se donnent ces documentaires d’obtenir l'image de tels ou tels événements ou personnalités politiques ou militaires au moment où on les nomme dans la narration conduit ainsi à évacuer sans vergogne les circonstances et l'époque dans lesquelles une image a pu être prise. La rareté des documents concernant certaines personnalités ou la nécessité de recourir à l'imagerie qui leur est attachée - pour obtenir un effet de reconnaissance immédiat décuple ces effets de décalages: pour «illustrer»le nom «Lénine» dans une évocation de la Révolution russe de 1917 , on ne peut recourir qu'à des images de I92I ou I922 prises lors d'un congrès de l'Internationale ou d'un autre événement, sans rapport avec l'épisode évoqué; quand on n’utilise pas les images mises en scène par Eisenstein dans Octobre (I927) pour le dixième anniversaire de l'événement... 
A cette inflation qui neutralise le contenu des images et en obère la valeur de document se sont opposées des pratiques de plusieurs types, émanant avant tout de cinéastes «d'art et d'essai» qui s'attachent soit à la singularité d'un document et en proposent l'exploration - comme Harun Farocki -, soit à sa matérialité, ses lacunes et finalement son opacité - comme Alexandre Sokourov, Yervant Gianikan et Angela Ricci Lucchi ou Serguéi Loznitsa. Dès les années I96o, le «recyclage» d'images trouvées, souvent d'actualité mais également de fiction, sans hiérarchie de valeur (films scientifiques, dessins animés, télévision, etc.), avait été pratiqué par des cinéastes expérimentaux avec des procédures de ré-appropriation diversifiées (Bruce Conner notamment). Ou par Guy Debord en France avec La Société du spectacle (I974). Depuis lors se sont imposées les catégories de «found footage» et de «réemploi», ou encore de «Mash-up» que la technologie numérique de l'audio-visuel a rendues aisées.

L'accessibilité accrue des fonds iconographiques permise et suscitée par ces nouvelles technologies (numérisation, indexation, consultation en ligne) a démultiplié et déplacé de nos jours ces stratégies formelles voire formalistes vers des démarches plus axées sur des enjeux de mémoire.

\section{Jean-Gabriel Périot}

Avant Une jeunesse allemande, Périot a réalisé plusieurs courts métrages qui s'inscrivaient dans un espace à la fois formaliste (images dédoublées, accélérées, saccadées, rythmées sur de la musique) et adoptant in fine une conclusion historico-réflexive, tels que Dies Irae, die illa (2005), Eût-elle été criminelle... (2006), 200 ooo fantômes (2007), Regarder les morts (2011), The Devil (2012). Dies Irae, die illa fait se succéder à grande vitesse en fondu enchaîné une série de perspectives axiales de routes puis de rues menant, lors des dernières images, au portail du camp d'Auschwitz. 200 oo० fantômes égrène des photographies de la vie paisible d'une ville que domine le dôme de son palais des expositions puis, après l'image noire d'une explosion, le même bâtiment, en ruine, resté seul, ou presque, debout dans une ville dévastée par la bombe atomique américaine. Rien n'est dit mais nous sommes à Hiroshima et la ville se reconstruit au gré de photographies successives autour du Dôme de Genbaku, ruine monumentalisée. Dans Eût-elle été criminelle, dès les premières images - une réunion de notables, puis des défilés de juges et autres autorités religieuses, civiles et militaires accélérées comme dans l'Entr'acte de Clair et Picabia -, 
retentit La Marseillaise, dont une mesure semble se suspendre en un son répétitif, le temps d'un montage ultra-rapide de soldats, d’images de guerre (canons, fusils, avions, cuirassés, etc.), de résistants, de ruines, du débarquement en Normandie, de la victoire, avant de reprendre son cours sur des images de liesse populaire, rondes et danses, défilés fêtant la libération. Puis les images sont recadrées, ralenties, répétées et, petit à petit, il apparaît que le «centre», d'abord absent, de cette joie collective tient en plusieurs scènes de tontes de femmes accusées de s'être compromises avec des soldats allemands pendant l'occupation. Les paroles de La Marseillaise retentissent alors jusqu'à la fin pendant que se succèdent ces scènes atroces qui avaient révulsé Michel Leiris ou Paul Eluard ${ }^{1}$. Comme dans les films brièvement évoqués ci-dessus, la construction de ce film est fondée sur un renversement qui ne peut que susciter l'effroi mais joue d'un seul ressort. D’après son auteur, ce film a son origine dans la découverte, par l'historienne Sylvie Lindeperg, de séquences tournées en 1944 après la Libération, non retenues par France Libre Actualités. L'intérêt de la démarche tenait alors, d’une part, à cette utilisation d'images non vues à l'époque (ce qui renvoie aux motivations de ceux qui montaient ces Actualités et jugeaient de ce qu'on pouvait montrer ou non ${ }^{2}$ ) et, d'autre part, à cette exploration du champ des images qui, en évitant le sujet central (la tonte), parcourait la foule en joie, inquiétant l'idée même de «libération ${ }^{3}$.

Evoquons pour finir The Devil pour sa proximité de construction avec Une jeunesse allemande: le film part d'une «série de portraits et de situations discriminatoires et violentes envers la population noire dans l'Amérique des années 6o» et se poursuit par «la prise en main du destin des Afro-Américains par eux-mêmes» puis par «la militarisation du mouvement, sa stigmatisation et sa répression par un Etat qui s'inquiète de la portée révolutionnaire et revendiquée du mouvement ${ }^{4}$.

\section{Une jeunesse allemande}

La structure générale d'Une jeunesse allemande offre les mêmes trois phases dans sa narration: portraits et situations d'affrontements politiques et idéologiques dans la société ouest-allemande; organisation des luttes, en particulier à Berlin-Ouest; «militarisation du mouvement, stigmatisation et répression par un Etat qui s’inquiète de la portée révolutionnaire et revendiquée du mouvement». La différence tient dans
1 «En ce temps-là, pour ne pas châtier les coupables, on maltraitait les filles. On alla même jusqu'à les tondre» (exergue de «Au rendez-vous allemand», Paul Eluard, I944).

2 Ce choix de ne pas utiliser ces séquences (on ne peut pas parler en l'espèce de censure) renvoie à la responsabilité des «monteurs» de France Libre Actualités et, au-delà sans doute, au statut qu'ont pris rapidement ces scènes dont la représentation donnée par le film ou la photographie incline le spectateur à la pitié envers les victimes plus qu'à l'assentiment du «châtiment».

3 La séquence de Persona (I966) où Bergman fait explorer à son héroïne la photo de l'enfant juif du ghetto de Varsovie s'apparente à cette démarche bien qu'elle procède a contrario: en recadrant, isolant, établissant de nouveaux rapports que la photographie entière laisse dans l'ombre ou ne permet pas de voir, le regard développe des "possibles» de l'image plutôt qu'elle ne les annule (voir F. Albera, "Temps d'arrêt», Retour d'y voir, $\mathrm{n}^{\circ} 6-7-8,20 \mathrm{I} 3$ ).

4 Les phrases entre guillemets appartiennent aux propres définitions de son film par le cinéaste. 
5 Elément extérieur au film et qui l'a sinon précédé du moins accompagné, Périot a établi une chronologie de 1965 à 1977 où il retient et détaille un certain nombre de faits et parfois en donne une appréciation. Cette chronologie pose évidemment le problème de ses choix en dépit de l'objectivité dont elle se prévaut. Il serait intéressant de la prendre en compte dans une perspective d'analyse «génétique» du film, mais trompeur dans la lecture qu'on en propose ici qui procède de son seul visionnement. Ce document a été rendu disponible à l'occasion de la présentation, au cinéma Utopia de Bordeaux le I5 mai 20I4, du «projet de long métrage documentaire de Jean-Gabriel Périot (20I0-20I4) en cours» avec une "projection d'une durée indéterminée d'un travail en cours, inachevé, forcément fragmentaire, qui sera accompagnée par son réalisateur». (http://monoquini.net/blog/index.php?/ en-cours/une-jeunesse-allemande/).

6 Le refus du commentaire omniscient est un parti pris du documentaire d'auteur encore qu'il puisse y avoir d'autres manières de l'appréhender, comme ont pu le faire Pasolini dans La rabbia (I963) ou Franco Fortini dans All'armi siam fascisti (I962), deux films de montage d'actualités ou d'archives avec un texte off continu de valeur plus littéraire que de commentaire. Sans parler de Chris Marker dans la quasi totalité de son œuvre.

7 Entretien dans Télérama, I5 octobre 20I5. On a pourtant publié depuis la sortie du film un texte programmatique explicitant bel et bien le point de vue du cinéaste, en tout cas à un moment de son travail sur ce film - étendu sur de nombreuses années (2006-2015) - intitulé «Une histoire visuelle: la Rote Armee Fraktion et ses images. Note d'intention» (dans Nicole Brenez et Isabelle Marinone, éd., Cinémas

l'accent mis lors de cette troisième phase sur l'isolement des activistes, la nature criminelle de leurs actions et leur échec les conduisant au suicide.

Ces trois phases suivent une chronologie historique que permettent de fixer de manière plus ou moins explicite les dates inscrites en surimpression de certains extraits de films ou d'émissions de télévision cités - ou internes à ces documents -, chronologie «objective» qui «double», sous-tend la chronologie du film lui-même retraçant l'évolution d'un certain nombre de personnages progressivement identifiés et que l'on suit jusqu’à leurs morts: Ulrike Meinhof, Holger Meins, Andreas Baader, Gudrun Ensslin notamment ${ }^{5}$.

L'une des difficultés que présente ce film tient à la grande hétérogénéité des matériaux utilisés: films mis en scène, films inachevés, exercices d'école, émissions de télévision, films d'amateurs, documentaires, etc. Le passage d'un fragment à l'autre, la juxtaposition ou la succession d'un son et d'un plan acquièrent dès lors une tout autre importance que dans un film dont le matériau archivistique est homogène. Celui-ci possède une certaine continuité qui fait passer ses raccourcis ou ses omissions pour des ellipses: l'univers diégétique qu'il instaure donne l'illusion de la cohérence. Ici la diégèse est à l'évidence construite quoique «objectivée» par la chronologie historique. L'analyse du film doit donc s'émanciper de cette naturalisation de la chronologie censée adopter celle de l'histoire (de la suite des événements) et doit s’interroger sur ce qui n’apparaît pas, y voir des lacunes ou des omissions plutôt que des ellipses ou des raccourcis.

Rompu à l'exercice du montage, puisqu'il a été monteur de films industriels notamment ou de documentaires techniques qu'il était chargé de «condenser» pour le Centre de création industrielle (CCI) du Centre Pompidou, le réalisateur a collecté un grand nombre de matériaux filmiques et télévisuels de diverses provenances pendant plusieurs années et il s'est donné une règle en les assemblant: celle de ne leur adjoindre aucuns "commentaires en voix off, ni interviews rétrospectives " ${ }^{6}$, voulant «surtout construire le film sans prescience, ni explication a posteriori de l'histoire. En la développant, dans son cours chronologique, en gardant la manière dont elle avait été vue, analysée ou racontée à l'époque, par les protagonistes ou les télévisions ${ }^{7}$.

libertaires: au service des forces de transgression et de révolte, Lille, Presses universi- taires du Septentrion, «Arts du spectacle Images et sons», 20I5, pp. 6I-70). 
C'est le risque qu'il a pris, c'est la gageure qu'il tient et c'est une des principales qualités qu’on a reconnues à son film. On a apprécié que les images soient livrées «brutes» et non filtrées par un point de vue, ni même la plupart du temps identifiées, datées, référencées. Lui-même revendiquant de n'avoir pas de point de vue sur son objet.

Ce parti pris peut, à l'inverse, lui être reproché si l'on se place d'un point de vue déontologique, archivistique. La seule critique approfondie de ce film parue en France, sous la signature de Daniel Sauvaget, le fait. L'auteur qui connaît bien le matériel utilisé déplore que le spectateur soit laissé dans l'ignorance de la provenance, du contexte, de la signification de ces sources ${ }^{8}$. C’est une position défendable: après le cinéma, la télévision nous a trop souvent accablés de ces montages sans principe où l'on mêle tout sans considération de l'identité des extraits et même de ceux qui y apparaissent, qu'on peut légitimement s'insurger contre la reconduction d'un tel brouillage référentiel dans un film « d'auteur» ${ }^{9}$.

Cependant, même en se situant en dehors de cette exigence d'historien attaché à la nature des documents utilisés - car Périot ne prétend pas être historien, tout au plus a-t-il formulé le projet, dans la note d'intention citée plus haut, de constituer une «histoire visuelle»-, les types de matériaux filmés qui constituent le «texte» de ce film ramènent sans cesse le spectateur à s'interroger sur leur identité, leur origine, leur nature. En effet si l'un des apports incontestables de Périot a été, dans la préparation de son film, de mettre à jour toute une «filmographie» oubliée ou occultée due aux protagonistes mêmes de l'épisode politique qu'il relate, on reste généralement dans l'ignorance du statut de ces différents extraits. En dehors des images de télévision (journaux télévisés, émissions), on a, d'une part, des films d'Ulrike Meinhof réalisés entre i965 et i970 pour la télévision (la Norddeutscher Rundfunk de Hambourg ${ }^{10}$ ), d'autre part, des films réalisés par les étudiants de l'école de cinéma de Berlin, la DFFB (dont certains feront partie de la Rote Armee Fraktion [RAF]), les uns documentant les événements (prises de parole, manifestations, répression policière), les autres entrant dans la catégorie de l'agit-prop, des ciné-tracts de type soit proclamatifs, soit satiriques, d'autres encore qui sont des documentaires sociaux (comme le film d'Holger Meins, Oskar Langenfeld [1966]). Ils n’ont évidemment pas connu la même audience et certains des films d'étudiants n’ont, sauf erreur, jamais été terminés ni montrés sinon à quelques personnes seulement. Leur rassemblement
8 Daniel Sauvaget, «Une jeunesse allemande», Jeune Cinéma, n 369-370, 2015, pp. $7^{-15}$.

9 Le problème n'est pas nouveau puisque Viktor Chklovski, à plusieurs reprises, fit ce reproche à Vertov comme à Choub: "Il faut débattre avec Choub, écrit-il, non pas du matériau mais de ses méthodes d'utilisation. Un fait privé de date s'esthétise et s'altère.» ("A propos du film d'Esther Choub», Novy Lef, $\mathrm{n}^{\circ} 8-9$, I927, traduit dans V. Chklovski, Textes sur le cinéma, Lausanne, L’Age d'Homme, 20II, p. 206).

10 Il s'agit de Arbeitsunfälle (Accidents du travail, diffusé le 24 mai I965), Arbeitsplatz und Stoppuhr (Usine et chronomètre, diffusé le 9 août I965), deux films consacrés au monde du travail, aux cadences, aux risques d'accidents, et Bambule ( $\mathrm{Mu}$ tinerie, 1969, interdit) tourné avec des pensionnaires d'un foyer pour jeunes femmes. 
sous la catégorie de «films de la RAF» est donc discutable, d'autant plus que bon nombre d'entre eux sont antérieurs à la constitution de ce groupe politique.

En outre tous ces films sont construits, développent un discours très articulé et bon nombre réfléchissent la question du cinéma, des images et des sons à faire, de la responsabilité sociale du cinéaste, etc. En extraire quelques secondes ou minutes en les considérant tous «à égalité» comme reflets, témoins, indices - comme le seraient des bandes d'actualités - est dès lors difficilement tenable.

Par ailleurs, on l'a dit, l'autre grand corpus d'images et de sons qu'a réuni Périot appartient à la production des grands médias, avant tout les télévisions allemandes, mais aussi française et suisse romande. Au projet initial (si l'on en croit la «note d'intention») d'envisager «Une histoire visuelle: la Rote Armee Fraktion et ses images» s'est substituée une confrontation entre deux productions d'images et de sons, celle de la télévision (porteuse du discours de l'Etat ouest-allemand) et celle des activistes politiques (qui le combattent). Or cette confrontation rencontre deux contradictions, l'une synchronique et l'autre diachronique: d'une part la production des activistes est hétérogène et entre en intersection avec la production télévisée (Meinhof réalise tous ses films pour la télévision, certains des films des étudiants sont cités ou repris par une télévision - on ne sait pas quand, il est vrai); d'autre part la confrontation cesse dès lors que les activistes - du moins ceux que le film retient comme ses personnages (Meinhof, Meins) - ne tournent plus en raison de la forme qu’a prise leur lutte politique (clandestinité, action directe). Dès lors le déroulement des événements jusqu'à la mort des protagonistes n'est plus documenté que par la production télévisuelle. Les activistes sont, de ce fait, privés de toute expression - bien qu'ils se soient exprimés sous différentes formes - et le point de vue que l'on en donne est celui de l'Etat fédéral (avec une présence croissante des dirigeants politiques de la coalition SPD-CDU au pouvoir) et des organes médiatiques «organiques» (presse Springer, télévisions).

\section{Corpus}

Cette disparité pose à vrai dire le problème du corpus que s'est donné Périot. En voulant mettre en opposition - et sans doute en miroir (la thèse sous-jacente de son film est celle de la «complémentarité» des 
deux adversaires ${ }^{11}$ ) - l'imagerie étatique et l'imagerie révolutionnaire, il néglige délibérément d'autres types de production cinématographique contemporaine, d'autres positionnements que celui des membres de la RAF (ou qui vont le devenir). Caractéristique à cet égard est l'utilisation qui est faite d'un entretien télévisé avec Alexandre Kluge dans le film. On lui demande longuement «comment un cinéaste qui essaie de raconter des histoires à l'aide d'images en mouvement peut, de nos jours, avec ses histoires, atteindre la société, l'émouvoir, éventuellement la toucher et, finalement même, la changer? Est-ce possible?» Suit un contrechamp sur Kluge, mais alors qu'il allait répondre on passe à l'inauguration de la DFFB, l'école de cinéma de Berlin, par Willy Brandt et on isole parmi les étudiants qui assistent à la cérémonie l'un d'entre eux, Holger Meins, dont on montre ensuite des extraits de films. Meins se substitue donc à Kluge via le discours laborieux et lénifiant de Brandt exprimant le dessein de l'industrie du cinéma allemand en crise de se relancer avec de jeunes artistes. Et la DFFB semble se réduire aux étudiants qui iront du côté de la RAF (le commentaire dit: «Mais les plus doués des étudiants se révèlent des rebelles de gauche... ${ }^{12}$ ). Pourtant Kluge dirige à cette même époque le département cinéma («Design cinématographique») de la Hochschule für Gestaltung d’Ulm (créée par Max Bill quelques années plus tôt sur le modèle du Bauhaus), né avant la DFFB, et de celui-ci sortiront aussi plusieurs cinéastes militants des causes politiques et sociales qui mobilisent alors une partie de la population d'Allemagne fédérale (et de Berlin-Ouest), à partir de i967. Kluge, de surcroît, participe au mouvement du nouveau cinéma allemand qui a vu le jour à Oberhausen en 1965.

La question se pose donc de savoir s'il n'a pas été possible de retrouver un seul film documentaire, analytique ou même militant du temps de la RAF qui n'émanât pas de celle-ci ou de ses partisans ou si on les a délibérément écartés. Sans rechercher l'exhaustivité, on peut citer Claudia von Alemann à Ulm - qui participe à des collectifs et tourne notamment Das ist nur der Anfang - Der Kampf geht weiter (1968-1969) -, Abisag Tüllmann, Hans-Jürgen Krahl, Erika Sulzer-Kleinemeier, Günther Hörmann à Francfort, qui couvrent les événements politiques et réalisent des films engagés dans les luttes universitaires, sociales et antiimpérialistes. On a d'ailleurs, lors de l'inauguration de la DFFB, quelques plans de coupe sur les étudiants qui montrent notamment Harun Farocki et Daniel Schmid. Farocki est ensuite plusieurs fois visible lors de
11 Topos dans les commentaires de l'époque de la part des adversaires de la RAF ou de leurs critiques «de gauche» (voir en particulier le numéro des Temps Modernes, $\mathrm{n}^{\circ}$ 396-397, juilletaoût 1979. «Allemagne fédérale: difficile démocratie»).

12 Dix-sept seront renvoyés en raison de leurs activités militantes (dont Meins, Farocki, Bitomsky notamment) et certains réadmis (comme Farocki). Le directeur des études était alors Erwin Leiser. 
Parmi les étudiants de l'Académie du cinéma de Berlin-Ouest inaugurée par Willy Brandt: Harun Farocki et Daniel Schmid

13 Die Wörter des Vorsitzenden, I967; Nicht Löschbares Feuer, 1969; Der Ärger mit den Bildern, I970; Moderaten in Fernsehen, I974.

14 «Tous les agents de la fonction publique sont tenus d'adhérer à l'ordre fondamental libéral et démocratique au sens de la Loi fondamentale, et de le protéger. Des menées hostiles à la Constitution représentent une violation de cet engagement.» («Déclaration commune de l'ancien chancelier fédéral Willy Brandt et du président du Conseil des Länder» du 28 janvier I972). Voir Peter Brückner et Alfred Krovoza, Staastfeind: Innerstaatliche Feinderklärung in der Bundesrepublik, Berlin, Wagenbach, I972 [Ennemis de l'Etat: mise à l'index de l'ennemi intérieur en RFA, Paris, La Pensée sauvage, I979. Préface de Michel Foucault]; Jacques Denis, Liberté d'opinion... Verboten. Les interdictions professionnelles en RFA, Paris, Editions sociales, 1976 , en particulier les «Documents et annexes» qui documentent le fichage et la «chasse aux sorcières» alors à l'œuvre. Voir aussi Daniel Vernet, «L'ordre règne en Allemagne fédérale. Démocratie et limitation des droits fondamentaux, des moyens efficaces pour assurer le calme et le conformisme», Le Monde diplomatique, avril 1976 et Sebastian Cobler, «RFA: l’Etat normal»», Les Temps Modernes, op. cit., pp. 50-88.

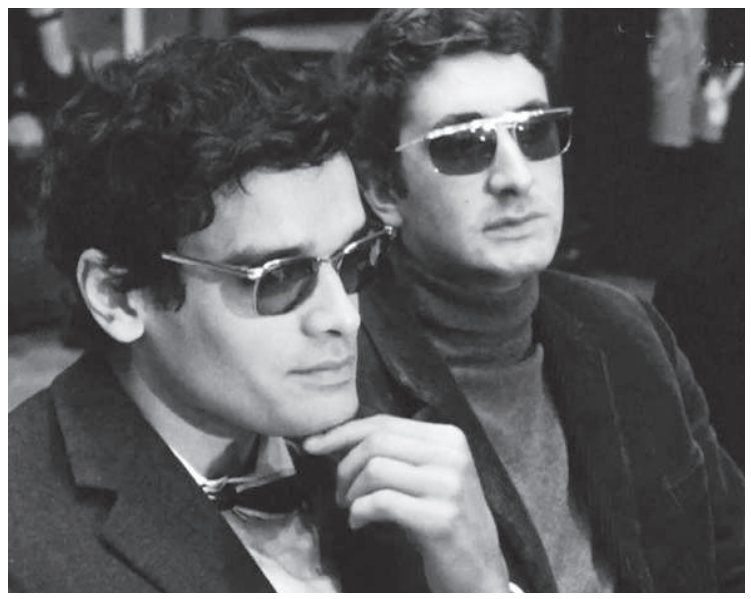

Une jeunesse allemande (Jean-Gabriel Périot, 2015)

manifestations ou d'assemblées mais il ne prend pas le chemin de Meins et de la RAF. Pourtant il tourne des films politiques dans cette période, absents du choix de Périot ${ }^{13}$ - comme Kluge (qui tourne In Gefahr und Größter not ist der Mittelweg der Tod en 1977).

On se pose, de même, la question de savoir s'il n'y eut aucune action politique, syndicale ou autre dans la RFA et à Berlin-Ouest du temps de la RAF qui ne répondît pas à l'analyse et à la praxis de cette dernière. Quoi qu'on pense des moyens d'action que crurent devoir adopter les membres de la RAF, leurs actions faisaient partie d'une situation d'ensemble qui semble s'évanouir dès lors que la RAF est fondée. Le narratif du film évoque les mouvements étudiants - emmenés par le SDS (association des étudiants socialistes) dans toutes les grandes villes de RFA -, les mouvements politiques opposés à ce qui est largement considéré par les manifestants comme une fascisation du régime (lois d'exception, écoutes téléphoniques, réarmement, rôle de la RFA dans l'OTAN, présence américaine en pleine guerre du Vietnam, etc.). Rappelons qu'à l'époque dont on parle, la politique des «interdits professionnels» (Berufsverbote) soumettent 850 ooo fonctionnaires ouest-allemands ou candidats à la fonction publique à des enquêtes secrètes et des interrogatoires amenant au renvoi de 4000 d'entre eux au nom du manque de «loyauté envers la constitution ${ }^{14}$. 
Les incarcérations successives des activistes du Mouvement du 2 juin (formé en $1967^{15}$ ) et de la Rote Armee Fraktion (1970) débutent en I968 et s'achèvent en I977. Les conditions de ces incarcérations ont été largement dénoncées en RFA comme au plan international (Amnesty International, Tribunal Russell) en raison de leur inhumanité (narcose forcée, isolement sensoriel ${ }^{16}$, absence de soins appropriés, brutalités physiques), de même les restrictions apportées aux droits de la défense (interdiction de plaider des avocats, arrestations de ceux-ci). Les prisonniers répondent à la violence carcérale par des grèves de la faim répétées. Le film eût pu évoquer ces aspects via des déclarations, ne serait-ce que celle de Jean-Paul Sartre se rendant à Stammheim pour rencontrer Baader et manifester sa solidarité avec les prisonniers soumis à ce régime carcéral. Nul doute qu’une télévision ou un film ait enregistré sa venue... Sans compter les actions de solidarité avec les avocats persécutés ${ }^{17}$, voire leurs déclarations qui furent en partie diffusées par la télévision puisque le chancelier fédéral Helmut Schmidt blâma les chaînes d'avoir laisser passer des extraits des déclarations à la presse des avocats de Baader, Ensslin et Raspe en grève de la faim - Heldmann, Schily, Oberwinder et Weidenhammer -, expliquant qu'ils sont sous écoute téléphonique.

Cette réduction aux «films de la $\mathrm{RAF}$ » comme seuls antagonistes aux médias amène le film à progressivement laisser toute la place à la télévision et donc à ceux qui l'utilisent pour s'adresser à l'opinion publique (les autorités politiques et leurs commentateurs) et à restreindre toutes les luttes politiques et sociales à l'affrontement RAF/RFA.

Le refus du point de vue (mais pas le refus du choix on l'a vu) prend le risque de laisser les paroles et les images ré-utilisées «prendre le pouvoir» au sein de ce film sans point de vue: car la parole analytique, l'analyse politique, auxquelles s'est refusé le réalisateur, sont très présentes dans la bande son. L'absence de commentaire off n'empêche pas que le commentaire in (ou plutôt in/off: il est l'off des extraits cités et donc l'in du film de Périot) ne se fasse entendre et puisse s'imposer par la persuasion qui en émane ou par l'absence de contradiction qui lui est apportée. C'est ainsi, pourrait-on dire, que le film commence sous le regard et l'analyse d'Ulrike Meinhof - via ses prestations télévisées et ses films - et s'achève sous ceux de Helmut Schmidt. La «démonstration» qu’opère le film étant qu'à une situation sociale et politique insupportable (le legs
15 Ce jour-là, à Berlin, un policier abat d'une balle un manifestant contre la visite du Chah d'Iran.

16 «Une privation complète et pendant une longue période de toutes les impressions sensorielles [...] produit un besoin intense d'impressions sensorielles et de mouvement corporel, une forte suggestibilité, des difficultés à penser, une impossibilité à se concentrer, un état dépressif, éventuellement des hallucinations...» (Dr Petri, médecin-chef de la prison du Land de Nordrhein-Westphalie, cité par Die Zeit du i ${ }^{\text {er }}$ mars I974 dans un article intitulé "Torture en RFA?», cité dans Textes des prisonniers de la "fraction armée rouge» et dernières lettres d'Ulrike Meinhof, Paris, Maspéro, «Cahiers libres » 337, I977, p. 23I).

17 Cf. Gilles Deleuze et Félix Guattari, «Le pire moyen de faire l'Europe», Le Monde, 2 novembre 1977 . 

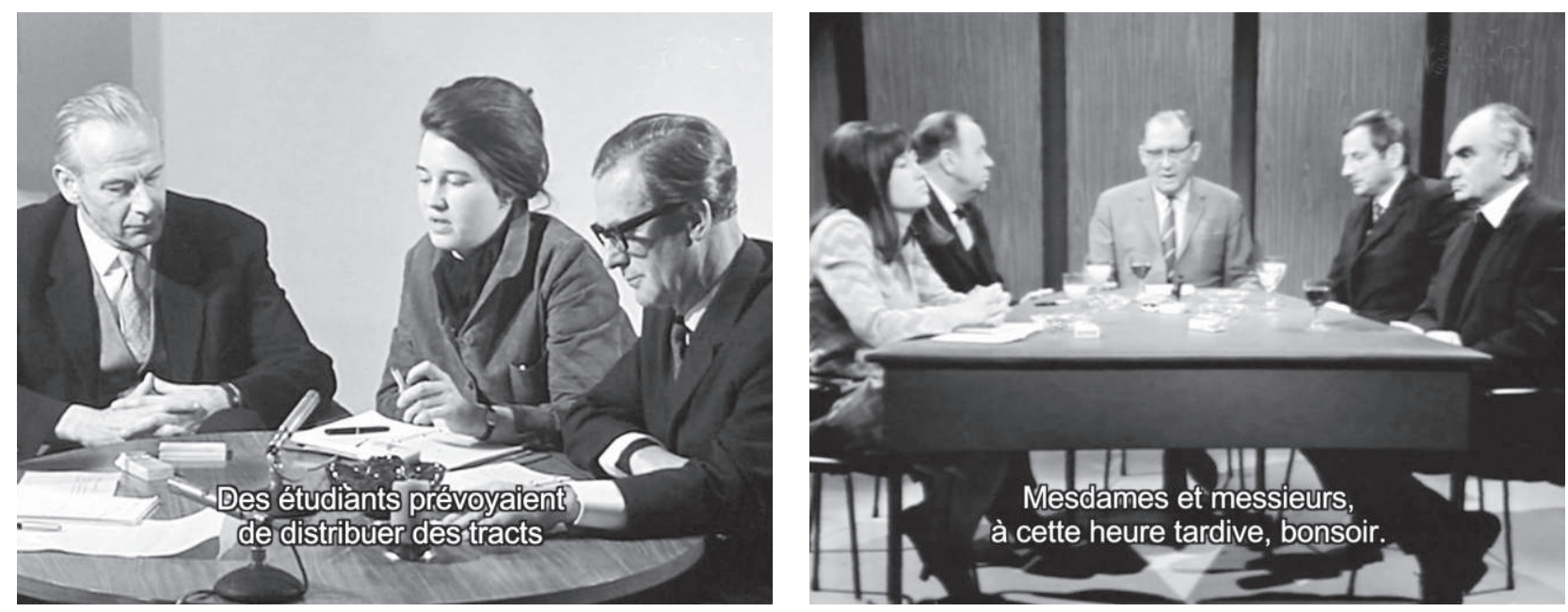

Ulrike Meinhof dans le dispositif télévisuel

du nazisme, le réarmement de la RFA sous la protection américaine, sa place dans le dispositif de l'OTAN, dans la guerre du Vietnam, la complicité avec des régimes oppressifs comme celui du Chah d'Iran, l'exploitation capitaliste dans les entreprises et l'autoritarisme d'une société qui a perdu toute crédibilité), la jeunesse oppose une légitime aspiration à un changement mais que les formes que prennent les luttes, dès lors que l'on passe à l'action directe, aux bombes, aux enlèvements, conduisent le mouvement à se réduire, s’isoler et se détruire lui-même. Or ce récit-là, s'il se «légitime» par la chronologie (que dans la troisième partie du film la seule télévision assure) et l'argumentaire des médias et de l'Etat ouestallemand, n'en est pas moins une construction ôtant progressivement la parole aux contestataires et les isolant de tout contexte local, national ou international conduisant à rendre «crédibles» les nombreux discours d'Helmut Schmidt et d'autres hommes politiques devant le Parlement ou à l'adresse de la population. Le constat que fait Ulrike Meinhof au début du film - dans des débats télévisés, des éditoriaux ou quelques films d'une «crise de l'autorité» adossée à un autoritarisme hérité du nazisme et exercé par des hommes qui lui appartinrent, suscitant la révolte de la jeunesse, ce constat se voit retourné dans les discours des hommes poli- 


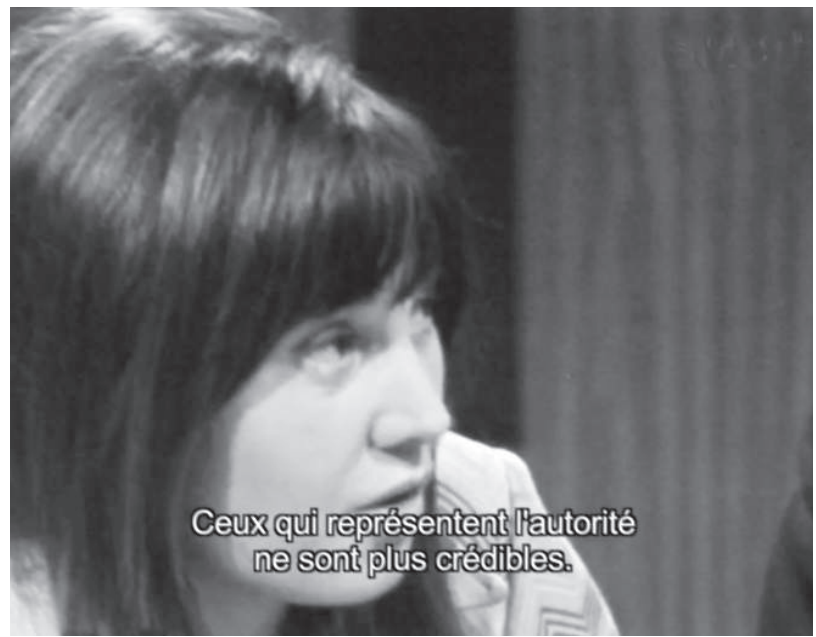

L'analyse de Meinhof domine la première partie du film

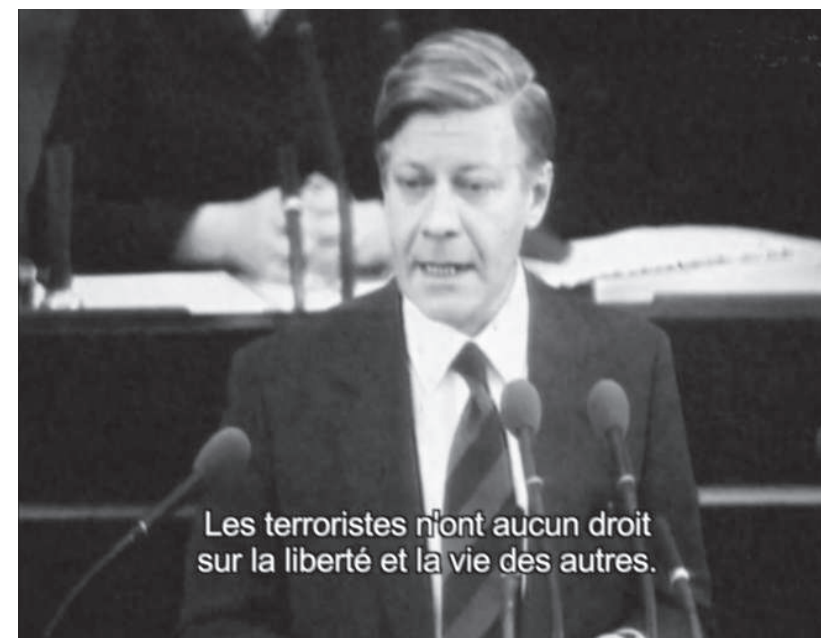

Les déclarations du chancelier Helmut Schmidt (SPD) dominent la dernière partie du film tiques du SPD et de la CDU (Schmidt, Kohl, Strauss - formant, à l'époque déjà, une coalition) qui traitent les membres de la RAF et les intellectuels qui veulent expliquer leur action de «nazis».

\section{Plan rapproché}

Cette structure en trois phases correspond en quelque sorte au film vu en «plan général», c'est son scénario. Il est important d'en saisir la logique - insatisfaction, révolte, violence, mort - et les conditions d'exposition: la focalisation sur quelques personnages isolant progressivement ces derniers du milieu qui est le leur et des mouvements collectifs auxquels ils participent. Le film met en scène des destins tragiques dont la mort (présentée comme suicidaire - grève de la faim, pendaison, balle de revolver ${ }^{18}$ ) est inéluctable. Ce choix narratif et dramaturgique isolant les membres de la RAF du contexte politique et social - national et international - où ils mènent leur combat, invalide celui-ci sans examen ni discussion. Il perd ses déterminants collectifs pour se résumer à un face à face avec les seules autorités (police, justice, grande presse, patronat) ramenées à quelques figures antagonistes (Springer, tel juge, tel responsable de la police, Schleyer le patron des
18 Tous les «suicides» ont été discutés et le refus des autorités de laisser pratiquer des autopsies, visiter les cellules (rapidement nettoyées) ou accéder aux moyens de surveillance (enregistrements, films), au témoignage des gardiens, etc. laisse perdurer la présomption de «liquidation» des prisonniers. 
patrons). Cette construction exprime donc le «point de vue» de ce film sans point de vue que la chronologie «objective» et le montage d'images et de sons «d'actualités» garantissent par la double naturalisation qu’ils supportent.

Si l'on examine maintenant le film en "plan rapproché», voire en "gros-plan», afin de repérer comment cette macro-structure se réfléchit dans le détail des assemblages d'extraits mis en œuvre, on observe alors que le retournement (de l'imputation de «nazisme») se joue à un niveau beaucoup plus restreint - «en gros plan» - dès le début du film. Examinons ce début en ayant à l'esprit qu'il se veut «sans commentaire» et laissant «parler les images»:

- Le prologue est emprunté à un film en couleur qui montre des doigts en très gros plan manipulant de la pellicule super-8 puis une table de montage tandis qu'on entend une voix s'interroger sur le fait de savoir s'il est possible de faire des films en Allemagne aujourd'hui, de faire des images - au sens de l'imagination.

- Des photos de caméras de divers types se succèdent, montrées à la suite, tenues par des mains.

- Retentit L'Internationale chantée en allemand sur l'image noir-blanc en très gros-plan, brève, d'une tourelle de caméra comportant trois objectifs qui vient se braquer sur le spectateur, une main faisant la mise au point sur la bague de l'un d'entre eux.

- Un plan plus large substitue à la caméra le barillet d'un pistolet (les trois objectifs coïncidant avec les logements des balles) braqué sur le spectateur, tenu en avant de lui par un tireur penché, visant, légèrement flou,
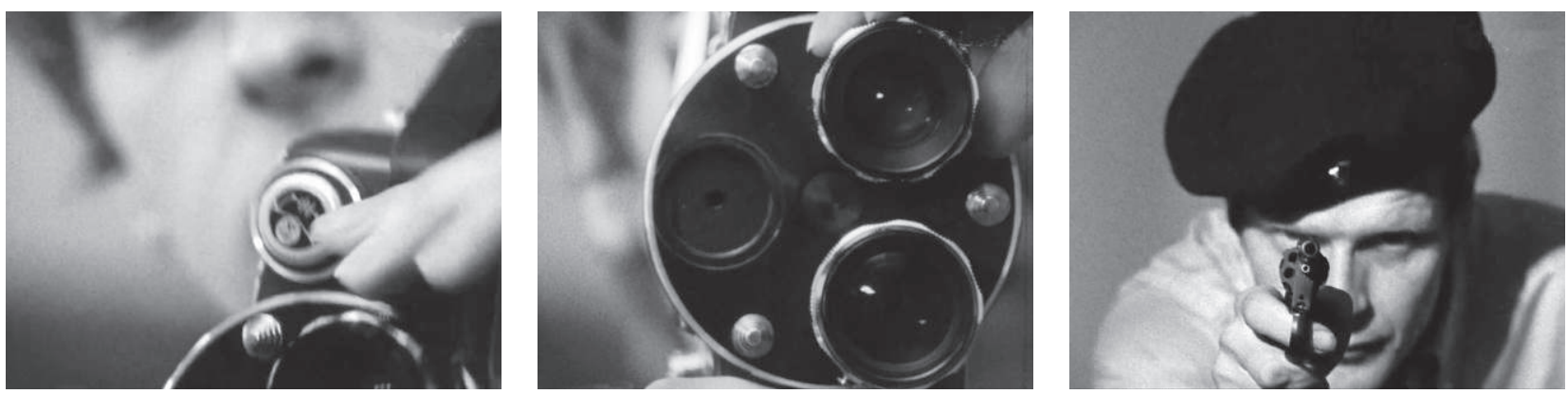

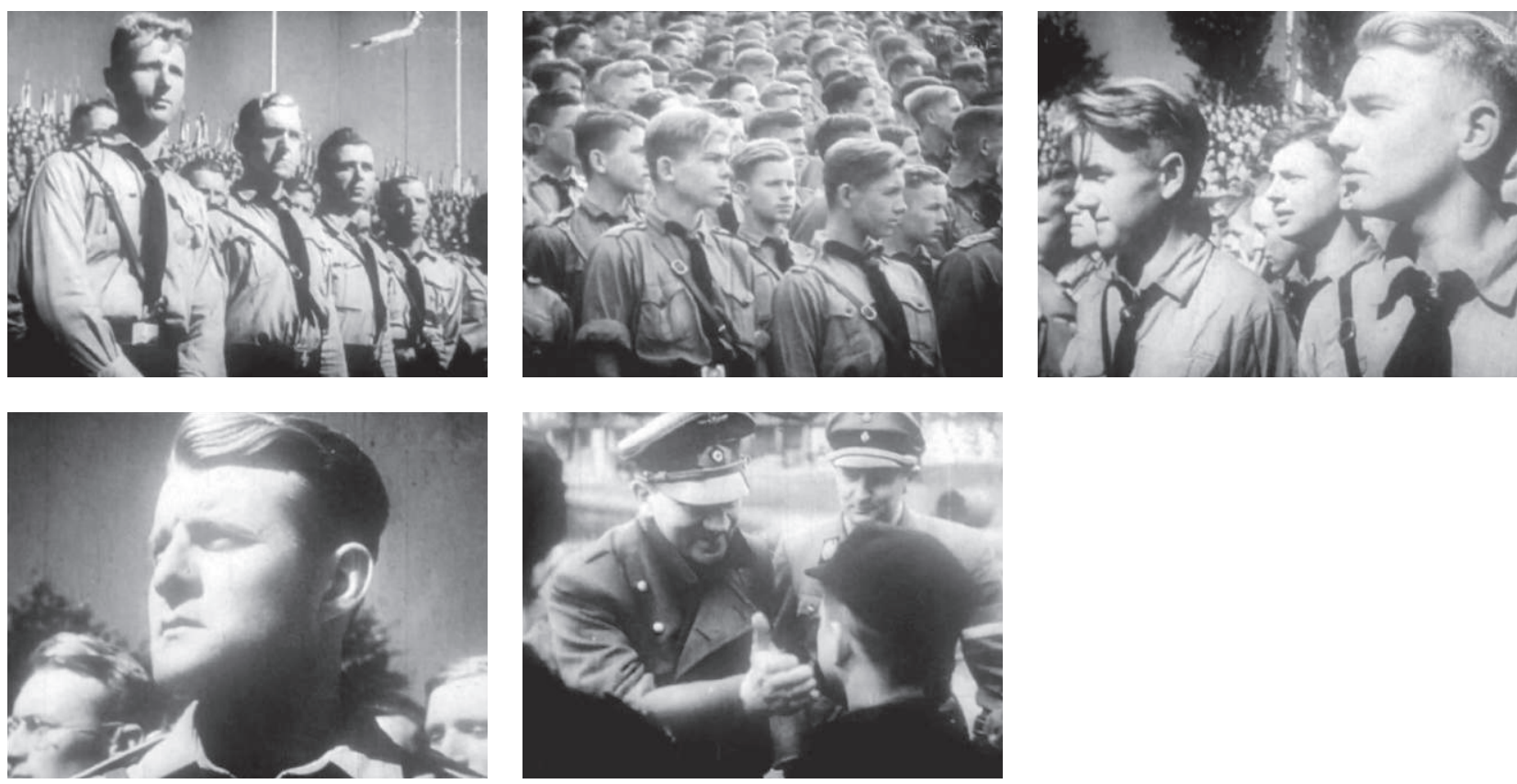

un jeune homme coiffé d'un béret ou d'une casquette. Plan fixe, long, qui sert de support au générique du film (c'est également l'affiche du film), sur le son de L'Internationale qui se poursuit.

- L'Internationale s'interrompt brusquement quand, au plan du tireur, se substitue un plan d'un groupe de jeunes gens de la Hitlerjugend alignés, immobiles, regardant devant eux, en uniforme (cravate, ceinturon, chemise militaire). Derrière les quatre jeunes gens une foule se devine et des drapeaux au loin. Puis un autre plan d'une foule de ces jeunes tous tournés vers la droite, tandis qu'une voix off énonce en français: «Il y a 20 ans la jeunesse allemande était au garde-à-vous... » Et un troisième où les jeunes gens regardent à gauche cette fois. Et deux autres plans encore, des gros plans.

- Un plan moyen de Hitler (de face) tapotant la joue d'un jeune garçon (de dos) et le commentaire off: «Dans l'Allemagne d'aujourd'hui les survivants préfèrent oublier mais leurs enfants ont du mal à comprendre ces images...» 
- Plan sur un autodafé de livres, la nuit. Commentaire: «Ils croient de nouveau à la culture et à l'intelligence et c'est sans doute ce qui, pour eux, est le plus symbolique de la folie nazie; il y a 30 ans, à Berlin, on brûlait les livres du poète Heinrich Heine ou de Bertolt Brecht...».

- Plan d'un homme d'une trentaine d'années debout devant une bibliothèque qui répond à un interviewer hors-champ et dit que «Seule la jeunesse est démocratique aujourd'hui en Allemagne. Il y a des nazis partout. L'Allemagne n'est pas stable... Il y a une hésitation entre la démocratie et l'idéologie... La seule chose où l'Allemagne est vraiment sûre, c'est dans la jeunesse...»

- Plan d'une croix gammée tracée manuellement à la peinture sur un mur occupant tout l'écran.

- Plan en plongée sur un groupe de jeunes et de passants qui discutent dans la rue à propos de cette croix gammée peinte sur un mur. L'un tient un micro. A droite, un homme portant une caméra sur son épaule. Une femme interroge: «Est-ce vous qui avez peint cette croix gammée sur le mur...»
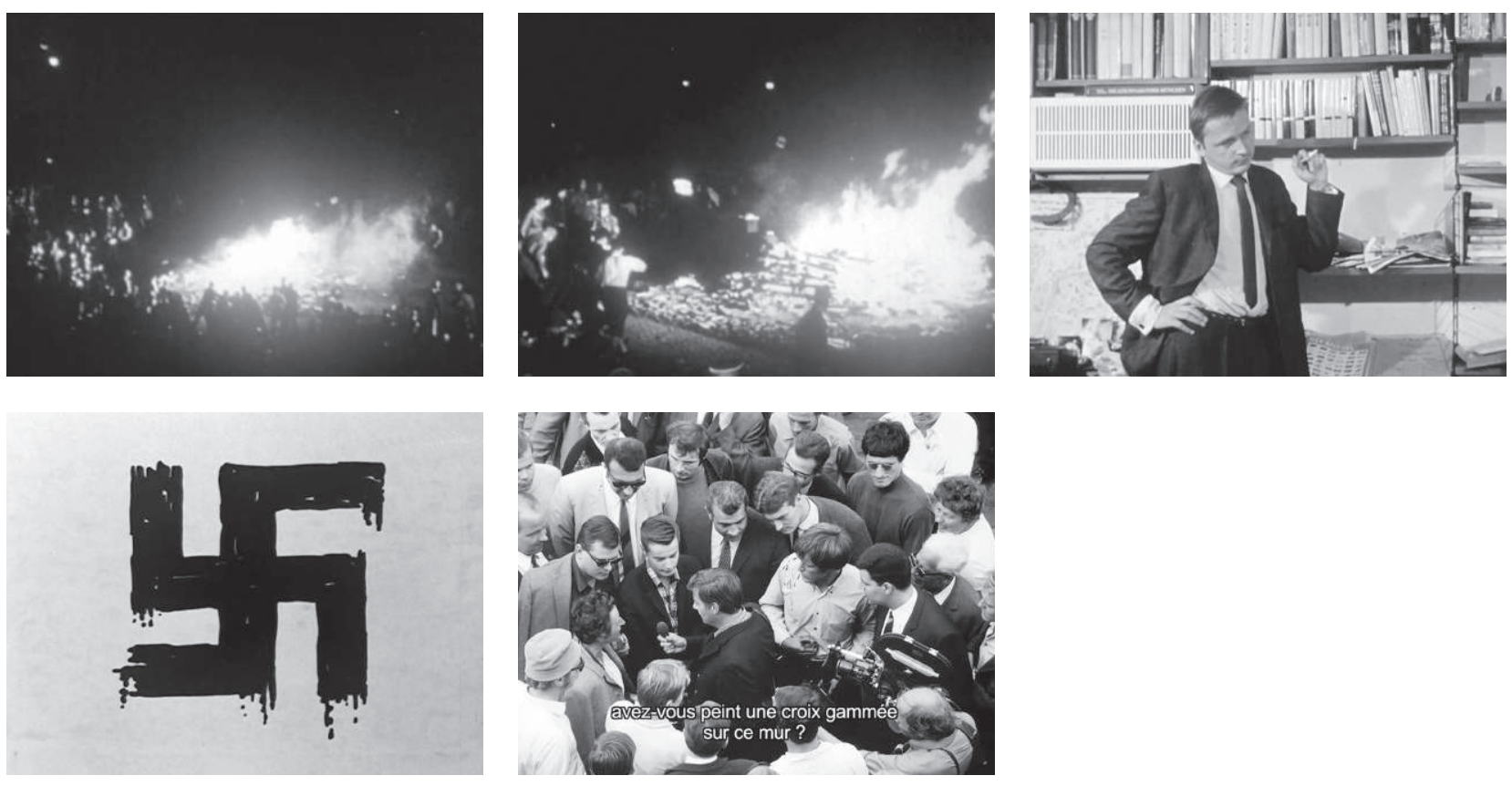

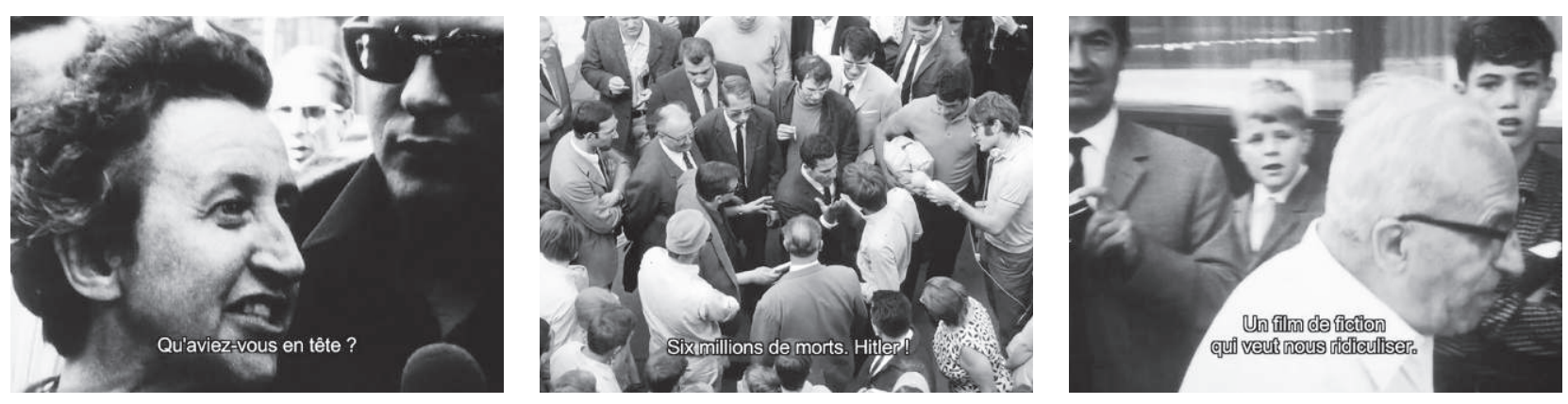

- Gros plan (issu de cette caméra portée) de cette femme qui continue: «Qu'aviez-vous en tête?»

- Retour au plan en plongée et apostrophe d'un jeune homme à l'endroit de celui que la femme interrogeait: «Six millions de morts! Je suis Juif!» Le jeune homme répond qu'il y a eu dix millions de morts en Allemagne. Et qu'il n'est pour rien dans la croix gammée... (on remarque des taches de peinture sur sa chemise blanche). Un quinquagénaire commence à parler: «A l'époque j'étais trop jeune...»

- Gros plan du quinquagénaire: - «...je devais obéir aux ordres». «Fallaitil obéir aux ordres?», dit un autre...

- Gros plan d’un autre homme sexagénaire: - «Sales gosses!» Une voix dit: «C’est un film de fiction». Il s'éloigne et revient en disant: - «Un film de fiction qui veut nous ridiculiser...» La caméra tourne autour de lui.

- Autre plan plus large avec le même homme qui poursuit: «...ça mérite une paire de baffes!» Il s'éloigne. Une voix: - «Grand-père, ce sont vos enfants!» Lui: «Mes enfants!». Il revient alors vers le groupe et gifle la caméra qui bascule, l’image devient floue.

On remarque d'emblée, dans cette suite d'une quinzaine de plans, combien nous sommes aux antipodes d'un film qui «laisserait» parler les images (et les sons) sans intervenir. L'organisation des matériaux par le montage est, au contraire, très délibérée et vise à pratiquer des rapprochements sinon des assimilations; en tout cas, elle instaure des mises en rapport lesquelles ne procèdent pas, pourrait-on dire, «de l'intérieur» des images et des sons mais de leur juxtaposition souvent brutale. 
19 L'image d'un tireur visant le public date de 1903 dans The Great Train Robbery de Porter. La caméra-fusil est entre autres un topos depuis le fusil photographique de Marey. Ici l'enchaînement des images et le chant spécifient très nettement le sens induit par le montage qui se rattache à Vertov comme à Godard. On aura plus loin L'Homme à la caméra (I929) sur le dépoli d'une table de montage et la proclamation par les étudiants que la DFFB est re-baptisée «Dsiga Wertow Akademie».
Le premier segment, où s'entend la voix [de Godard], est en couleur et appartient manifestement à un film sinon de fiction en tout cas très discursif, introduisant une réflexion sur l'Allemagne et les images, l'Allemagne et l'imagination. On ne nous dit pas quel est ce film. Selon la connaissance préalable qu'on peut avoir, on reconnaîtra ou non Das Kleine Godard de Hans Helmut Prinzler (I978), mais abstenons-nous d'en tenir compte puisque le film omet cette information. Cependant ce segment introduit le thème du cinéma, des images (qu'est-ce qu'une image? quelles images faire? à quelles fins?) qui traverse le film de Périot puis disparaît complètement. Il connaît un premier rebond dans le second plan, en noir et blanc, avec la caméra braquée sur le spectateur puis le jeune homme au pistolet visant le spectateur. Le son de L'Internationale inscrit cette image dans la mouvance du cinéma militant, le passage de la caméra au pistolet indique que l'on appréhende la caméra comme une arme et qu'on lui substitue une arme ${ }^{19}$.

Avec le passage cut sur les jeunes nazis au garde-à-vous, la consécutivité induit nolens volens un rapport d'analogie entre le jeune tireur gauchiste et les jeunes hitlériens. Les sèmes de détermination (fixité du regard, rigidité corporelle, béret ou casquette noire), para-militarisme, violence voire volonté de tuer se distribuent dans les deux segments successifs.

L'arrivée de Hitler tapotant la joue d'un enfant (qui n'est pas un jeune nazi - on a changé de film -, mais un enfant «quelconque») et la voix off disant que «les survivants préfèrent oublier mais la jeunesse ne comprend pas...» introduisent une contradiction entre jeunes et vieux (on dit survivants, terme ambigu dans la mesure où il n'est en général pas utilisé pour désigner les Allemands ayant «survécu» à la guerre mais les survivants des camps et en particulier d'extermination). Mais les «survivants » sont les jeunes des images.

Le segment suivant est d'une tout autre nature puisqu'il s'agit de l'interview d'un homme qui commente en journaliste, sociologue, politologue ou homme politique - on ne le sait pas - la situation allemande des années I960 au prisme de cette même opposition jeunes/vieux: la démocratie est du côté de la jeunesse, les adultes sont discrédités par leur compromission avec le nazisme.

Or le plan général suivant, pris dans la rue (mais manifestement organisé lui aussi voire mis en scène: deux caméras dont l'une surplombe 
la scène et une autre parmi le groupe de passants) rejoue la différence entre vieux qui furent jeunes sous Hitler et jeunes de i96o autour d'une croix gammée tracée sur un mur et qu’on a affichée auparavant plein cadre sur l'écran, en silence, comme en «conclusion» des propos de l'homme interviewé. Or ces jeunes et ces vieux des années I960 sont eux-mêmes divisés par cette même division qui était placée au plan générationnel et qui se révèle traverser les générations: la femme s’indigne de cette croix gammée, son interlocuteur, soupçonné de l'avoir tracée, est un jeune qui s'en défend (je n'y suis pour rien). Un Juif (jeune) s'oppose à ce même jeune supposé fascisant, une «vieille» s'indigne de ce retour du nazisme et un «vieux» se réfugie derrière le stéréotype de l'obéissance tandis qu'un autre gifle la caméra sans qu'on sache contre quoi il s'insurge: l'assimilation des jeunes (mais lesquels?) à «ses» enfants? Le fait même d'un tournage de fiction visant à «nous» ridiculiser?

Tout ce début peut être envisagé sur deux niveaux: celui de l'assemblage des extraits choisis par Périot dans son corpus (ici: [das Kleine Godard] - caméra-pistolet - Hitler Junge/Hitler à l'enfant/autodafé interview de l'homme - croix gammée/groupe de gens/gifle-caméra) et le montage interne à chaque extrait: est-il le montage tel qu'il se trouve dans l'extrait cité, choisi? A-t-il été retravaillé par Périot? On ne peut pas le savoir, sinon en se référant à une interview où le cinéaste déclare avoir respecté les raccords originaux des extraits. Mais passe-t-on, dans sa «source», des jeunesses hitlériennes à Hitler tapotant la joue de l'enfant et aux autodafés? Et quelle est la source? Est-ce l'émission de télévision (ou le film) sur l'Allemagne - d'évidence française puisque le commentaire est dans cette langue - qui pratique ce montage ou est-ce le film de propagande nazi, source de la source? S’instaure, on le voit, un jeu de miroirs infinis qui ne permet pas d'attribuer à une instance énonciatrice le geste de la mise en rapport. Mais là aussi l'image "parle d'elle-même» ou plutôt le montage nazi, par exemple, ou le montage du documentaire utilisant un ou des films nazis qui eux-mêmes, etc. Par conséquent le montage de Périot est en partie celui d'autres que lui qui produisent des effets qu'il cite sans qu'on sache s'il les assume (ainsi le passage du plan en plongée et du gros-plan sur la dame ou le monsieur indigné qui relèvent d'un découpage de fiction). Avec toute la bonne volonté du monde on doit avouer ne pas savoir ce qui s'énonce dans ce film à ce niveau-là ou plutôt - parce que «ça parle», c'est sûr - qui l'énonce. Passer 
20 Doit-on envisager que ce montage puisse vouloir signifier: «feu sur les hitlériens!»? Ce serait au mieux maladresse car ici, compte tenu des positions respectives des différents personnages, l'effet «champ/contrechamp» ne peut guère fonctionner, c'est le spectateur qui était visé non un hors-champ que les jeunes hitlériens viendraient combler. de L'Internationale aux jeunes hitlériens n'est pourtant pas bénin (Cf. $\mathrm{La}$ Marseillaise dans Eût-elle été criminelle...): le chant accompagne, soutient et dynamise le plan du tireur et, quand il s'arrête net, on débouche sur des jeunes nazis. Le tireur supposé gauchiste se voit dès lors assimilé aux jeunes hitlériens ${ }^{20}$.

On pourrait examiner tout le film de cette façon, pour y relever tout au long de telles ambiguïtés et de tels rapprochements ou analogies subreptices.

Ne citons que deux autres exemples liés aux premières apparitions d'Ulrike Meinhof. Le premier débute avec l'image d'une assemblée d'étudiants tirée d'une émission de télévision. Le commentaire évoque l'agitation dans les universités et stigmatise les «casseurs» qui «terrorisent», insultent les professeurs, distribuent des tracts. S’inscrit un lieu et une date en surimpression: "Berlin 1965" (de qui émanet-elle?). Les sèmes de jeunesse et de violence, qui avaient été introduits d'emblée on l'a vu, caractérisent également ici les propos et partiellement les images car cette violence, qui est du côté des «jeunes», des étudiants, pourrait faire écho aux autodafés du III ${ }^{\mathrm{e}}$ Reich - on ne brûle pas Heine et Brecht, mais on n'en bouscule pas moins les professeurs, on met à mal la culture (à laquelle on disait tout à l'heure que les jeunes

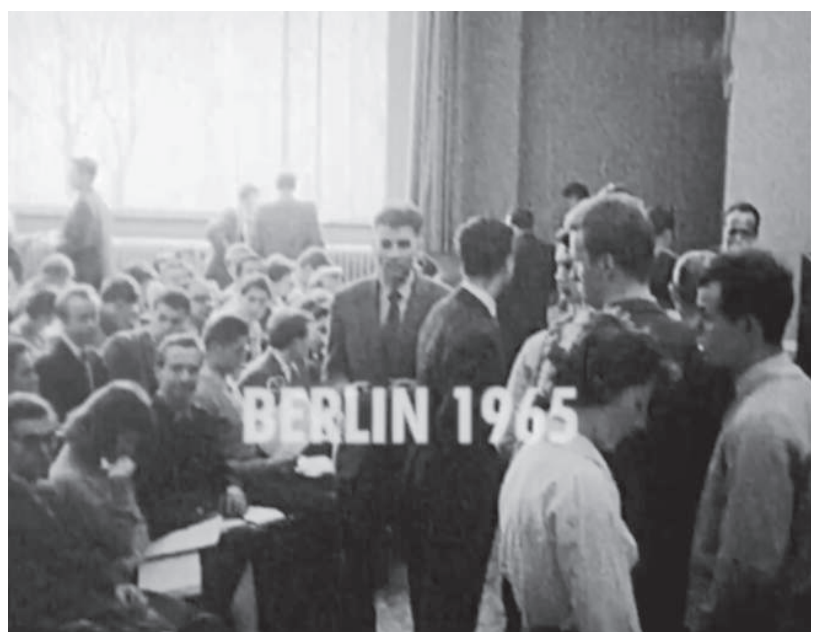


croyaient) - mais elle n'est pas montrée. Sur des images paisibles d'étudiants entrant dans leurs salles de cours, cheminant dans les couloirs ou à l'entrée des bâtiments, le commentateur dit: «On ne peut pas montrer ces images [de violence] car elles n’ont pas été filmées». Meinhof à qui on donne ensuite la parole prend le parti des étudiants puis on fait son portrait sur son image arrêtée. On passe ensuite à un autre débat où elle intervient, dont le titre est «Le Déclin de l'autorité». Mais avant qu'elle ne prenne la parole et juste après que le meneur de jeu l'eut brièvement présentée, sont insérés des plans en couleur de mer et de plage avec une voix off féminine censée exprimer les pensées de Meinhof (quand? où?) qui jette un doute sur ce qu'elle peut faire et dire dans la presse et à la télévision car on lui fait jouer un rôle de guignol: en l'occurrence, dire avec le sourire des choses qui sont vitales pour elle et pour nous ${ }^{21}$. Ainsi avant même qu'elle n'exprime son analyse des événements - qui se révélera très convaincante (s'agissant du doublet perte d'autorité et autoritarisme) -, on fragilise son personnage et son discours. On «annonce» en quelque sorte sa fuite en avant dans l'action et son «suicide». En revanche, rien ne viendra fragiliser la parole étatique réagissant aux attentats de la RAF.

Le film s'achève sur deux extraits de Deutschland im Herbst (L'Allemagne en automne), film collectif réalisé par Fassbinder, Kluge, Reitz, Schlöndorff, etc., en I978, qui tentait de réagir juste après l'enlèvement et l'assassinat de Hans-Martin Schleyer, le «patron des patrons» allemand, suivi des «suicides» de trois membres du groupe BaaderMeinhof en prison. Les deux moments de la contribution de Fassbinder sont l'un où, au téléphone, le cinéaste pleure et se désespère de ce qui vient de se passer (la mort des prisonniers de Stammheim) et l'autre où il met en scène un dialogue avec sa mère à qui il fait jouer le rôle de la réactionnaire qui veut de l'ordre. Comme dans le premier plan avec Godard, on ne nomme pas Fassbinder et on laisse planer le doute sur l'authenticité du dialogue qui semble pris sur le vif alors qu'il est mis en scène.

Il ne s'agit évidemment pas de contester à Périot le regard qu’il porte sur cet épisode historique, mais d'interroger les moyens qu'il se donne pour l'exprimer. Au sein d'un film qui introduit d'emblée un questionnement sur l'image et cite nombre de films qui se posent pour eux-mêmes ce problème, cela semble la moindre des choses.
21 Plans empruntés à Ulrike Marie Meinhof (1994), film de Timon Koulmasis, proche de Meinhof, amie de ses parents et mère de sa meilleure amie ( L'Histoire est écrite par les vainqueurs, c'est un lieu commun. Mais on n'est pas obligé de l'accepter telle quelle»). 
22 Jean Genet, Préface à Textes des prisonniers de la "fraction armée rouge», op. cit., p. I4 et p. I2. On peut rappeler que dans ces mêmes années, Jean-Marie Straub met en sous-titre à son premier long métrage, Nicht Versöhnt (1965) la formule de Brecht: «Seule la violence aide là où la violence règne.»

23 Félix Guattari, "Comme un écho de la mélancolie collective», Les Temps Modernes, $\mathrm{n}^{\circ}$ 396-397, juillet-août I979, p. $4 \mathrm{I} 6$.
Enfin, il faut bien le dire, ce film, dans le contexte actuel, prend une certaine actualité en nous renvoyant d'une part aux luttes politiques en RFA dans les années I960-I970 et à l'analyse qu'en faisaient certains militants (comme Meinhof et Baader) - entée sur l'internationalisation de l'économie (sous domination américaine) et sur le rôle dévolu à la social-démocratie européenne pour l'imposer - et, d’autre part, au type de réponse institutionnelle donnée à ces luttes - état d'urgence, lois d'exception, contrôle généralisé, conjonction des médias et du pouvoir, coalition «gauche-droite» (PDS-CDU) verrouillant le mécanisme de la démocratie parlementaire. Ce sont un certain nombre des problèmes que l'on retrouve aujourd'hui dans les sociétés européennes et américaines confrontées à une autre sorte de «terrorisme» et qui apportent des réponses du même type dont l'une des caractéristiques est d'étendre à l'ensemble de la société des procédures de contrôle, d'intrusion, d'intimidation, d'encourager la délation et de suspendre un certain nombre de «garanties» juridiques dont se prévalaient jusqu'ici les régimes politiques se réclamant de la «démocratie libérale».

Les membres de la RAF parlaient de «militarisation» de la société et de «nouveau fascisme» et, sur cette base, développèrent une stratégie de lutte armée (guérilla urbaine), opposant la «violence révolutionnaire» à ce que Jean Genet a appelé, à l'époque, la brutalité étatique: «Toute la «déclaration d'Ulrike pour la libération d'Andreas au procès de BerlinMoabit > dit très bien, d'une façon explicite, que c'est la brutalité même de la société allemande qui a rendu nécessaire la violence de la $\langle\mathrm{RAF}\rangle$ », écrit-il; et: «toute la violence spontanée de la vie continuée par la violence des révolutionnaires sera tout juste suffisante pour faire échec à la brutalité organisée.» ${ }^{22}$ On doit certes s'interroger sur le type de réponse apporté à cette brutalité par la RAF mais plus encore sur l'instrumentalisation qu'en ont faite les autorités politiques. Félix Guattari, à propos de L'Allemagne en automne, justement, disait qu'à son avis la RAF avait sous-estimé la place des «affects collectifs pétris et véhiculés par les mass-média» et s'était, pour cela, isolée. Il relevait enfin que «l'immense charge émotionnelle qui est associée au 〈phénomène terroriste〉 est devenue une donnée fondamentale des stratégies politiques actuelles ${ }^{23}$. Le film de Périot, en occultant cette montée de la brutalité étatique et son exaltation médiatique, ne nous permet pas d'appréhender, d'une part, les conditions d'émergence de ce recours à la lutte armée, ni, d'autre 
part, la place qu'occupe désormais le «phénomène terroriste» dans les politiques des régimes dits de «démocratie libérale». La perspective tracée par Guattari vient en effet inscrire le moment présent dans un mouvement de fond des sociétés occidentales et nous met en face de la labilité de la notion de «terrorisme» dès lors qu’on prétend «défendre la société».

On peut regretter que le film de Périot, se rendant captif des médias, en ait prolongé les effets. 